Check for updates

Cite this: Mater. Chem. Front., $2019,3,2128$

Received 18th June 2019, Accepted 19th August 2019

DOI: $10.1039 / c 9 q m 00395 a$

rsc.li/frontiers-materials

\title{
Flexible control of excited state transition under pressure/temperature: distinct stimuli-responsive behaviours of two ESIPT polymorphs $\dagger$
}

\author{
Aisen Li, ${ }^{a b}$ Hao Liu, ${ }^{a}$ Chongping Song, ${ }^{a}$ Yijia Geng, ${ }^{a}$ Shuping $X u, D^{a}$ \\ Hongyu Zhang, (D) ${ }^{a}$ Houyu Zhang, (D) ${ }^{a}$ Haining Cui ${ }^{b}$ and Weiqing $X u\left(D\right.$ ) ${ }^{2}$
}

\begin{abstract}
Realizing fexible and tunable emission conversion in organic solid-state luminescent materials is always fascinating. Herein, we successfully achieved fexible photochemical conversion under pressure and temperature on two types of 4-methyl-2-(5-[4-dimethylaminophenyl]-1H-pyrazole-3-yl)phenol (4MPP) single crystals with distinct aggregation form and geometry showing as green (GC) and blue-violet (BVC). These conversions were studied in detail by fluoresence and Raman spectroscopies, followed with Hirshfeld surface analysis, and this study provides a significant material system for understanding the mechanisms of structural conversion between normal emission ( $\left.E^{\star}\right)$ and excited state intramolecular proton transfer (ESIPT, $K^{\star}$ ) comprehensively under pressure and temperature. Owing to having a proton transferred tautomer, BVC exhibits remarkable changes in its emission feature with pressure increasing to $4.79 \mathrm{GPa}$, along with the blue-shift and fluorescence enhancement according to a conversion from $E^{*}$ to $K^{*}$. This compression-induced conversion is proved to be caused by the contraction of the intermolecular distances, the increase of molecular planarization degree, and the enhanced $\mathrm{O}-\mathrm{H} \cdots \mathrm{N}$ intramolecular interaction, along with the reduced distance of $\mathrm{H} \cdots \mathrm{N}$ and the elongation of the bond length of $\mathrm{O}-\mathrm{H}$. Differently, GC shows rapidly weakened fluorescence and slight blue-shift at a relatively lower pressure (1.01 GPa) based on its more restricted structure. Moreover, GC can realize an excited state transition from $K^{*}$ to $E^{*}$ when lowering its temperature. BVC experiences an abrupt phase transformation to $\mathrm{GC}$ at a high temperature of $190{ }^{\circ} \mathrm{C}$. This work discloses the photo-physical properties and stimuli-responsive behaviours of two 4MPP polymorphs, which provides deep insight into the relationship between molecular structure and photoluminescent nature, especially in the crystals with the ESIPT property.
\end{abstract}

\section{Introduction}

Organic solid-state luminescent materials have always been a hot topic due to their wide applications in the fields of organic optoelectronic devices and fluorescent sensors. ${ }^{1-4}$ Materials with excited-state intramolecular proton transfer (ESIPT) fluorophores have attracted considerable attention because of their remarkable properties such as a large Stokes shift, ${ }^{5}$ dual emission, ${ }^{5}$

\footnotetext{
${ }^{a}$ State Key Laboratory of Supramolecular Structure and Materials, College of Chemistry, Jilin University, Changchun 130012, P. R. China. E-mail: xuwq@jlu.edu.cn

${ }^{b}$ College of Physics, Jilin University, Changchun 130012, P. R. China

$\dagger$ Electronic supplementary information (ESI) available: Stacking structure, summary of intramolecular and intermolecular interactions, absorption spectra of GC and BVC, fluorescence spectra of BVC, Raman spectra under hydrostatic pressure, the analysis of theoretical results, temperature-dependent fluorescent photos and spectra, and recovery feature. CCDC 1576917 and 1576929. For ESI and crystallographic data in CIF or other electronic format see DOI: 10.1039/c9qm00395a
}

ultrafast process, ${ }^{6,7}$ and spectral sensitivity to surrounding media. ${ }^{8}$ The ESIPT upon photoexcitation facilitates the fluorescence emission process of chromophores via avoiding the selfabsorption quenching. Typical ESIPT molecules consist of proton donors and acceptors getting close enough and necessary intramolecular hydrogen bonds. ${ }^{9}$ Interestingly, ESIPT is a photochemical process, which produces a tautomer with an electronic structure different from the original excited form. ${ }^{10-12}$ The phototautomerisation process between the enol $\left(\mathrm{E}^{*}\right)$-keto $\left(\mathrm{K}^{*}\right)$ is realized through a four-level photo-cycle $\left(\mathrm{E}-\mathrm{E}^{*}-\mathrm{K}^{*}-\mathrm{K}-\mathrm{E}\right)$. In order to achieve multi-colour regulation of solid state emissions for ESIPT molecules, different strategies have been exploited, such as (i) controlling the mode of molecular packing, ${ }^{13}$ (ii) aggregation-induced emission (AIE), (iii) restriction of intramolecular rotation (RIR), (iv) torsion/planarity of enol conformers, etc. ${ }^{5,14-16}$ Accordingly, requirements become much stricter for the molecular design, organic synthesis of ESIPT molecules and cultivation of a polycrystalline phase. In fact, it is difficult to 
realize the emission conversion between $\mathrm{E}^{*}$ and $\mathrm{K}^{*}$ in a crystal phase.

Generally, the ESIPT process is easily affected by microenvironments around molecules because it needs a transient change. ${ }^{17}$ So slight changes in the intermolecular interaction or the molecular geometry easily impact the ESIPT emission via interfering with the proton transfer process, leading to remarkable changes in photo-physical properties. ${ }^{18,19}$ Therefore, stimulating ESIPT fluorophores by external elements is another feasible way of tuning the photo-physical behaviours, as well as achieving ESIPT-based sensing systems.

External stimuli, such as pressure and temperature, are two of the most common natural stimuli leading to completely different mechanisms. ${ }^{20}$ Temperature is an important environmental factor for regulating the photo-physical properties of various materials including ESIPT materials. ${ }^{21}$ Sekiya and co-workers reported an ESIPT molecule, 2-(2'-hydroxyphenyl)benzimidazole (HPBI) and found that the electronic spectra of the two crystal forms are significantly different at temperatures of $77-298 \mathrm{~K}^{22}$ Different from temperature, pressure can change the packing modes of molecules in crystals. Materials that can translate pressure impacts into changes in photo-physical properties are usually defined as piezochromic luminescent materials (PLMs). ${ }^{23,24}$ PLMs attract considerable interest due to their desirable properties and unique characteristics, allowing potential applications in memory devices, sensors and switches, etc. ${ }^{25,26}$ To date, the mechanisms of the piezochromic luminescence have been well investigated. Many mechanisms have been proposed to answer for various piezochromic phenomena, e.g., phase transformation, excimer formation and pressure-induced chemical changes under mechanical grinding, etc. ${ }^{27-29}$ Precise and gradient pressure control is welcome for the studies of PLMs. The shearing force attained from grinding is anisotropic and easy to manipulate, but uncontrollable and limited. In contrast, a hydrostatic pressure method produced from the diamond anvil cell (DAC) that can accurately and controllably provide an isotropic pressure as high as GPa becomes an impactful technique for investigating the PLMs. ${ }^{30,31}$ Under such a high pressure, many types of conversions on the packing ways of molecules and the luminous states can be realized, for instance, the transformation of polymorphs, ${ }^{32}$ the switching of the intramolecular charge transfer (ICT) process, ${ }^{33}$ the emission enhancement from the rehybridization of the nitrogen atom, ${ }^{34}$ and the separation of the hybridized local and charge transfer (HLCT) excited state. ${ }^{35}$ Though their pathways are different, most of them undertake reversible colour changes during the compression-decompression process. $^{36}$ So far, to our best knowledge, there are few reports on the pressure-dependent ESIPT mechanism, and the effects of pressure and/or temperature parameters on the fluorescence properties of these ESIPT fluorophores have not been well investigated in detail.

In this study, two types of 4-methyl-2-(5-[4-dimethylaminophenyl]-1H-pyrazole-3-yl)phenol (4MPP) single crystals showing as green crystal (GC) and blue-violet crystal (BVC) as in Scheme 1 were chosen, for detailed investigations of pressureand temperature-dependent photo-physical properties. GC and BVC exhibit distinguishable intramolecular conformations and

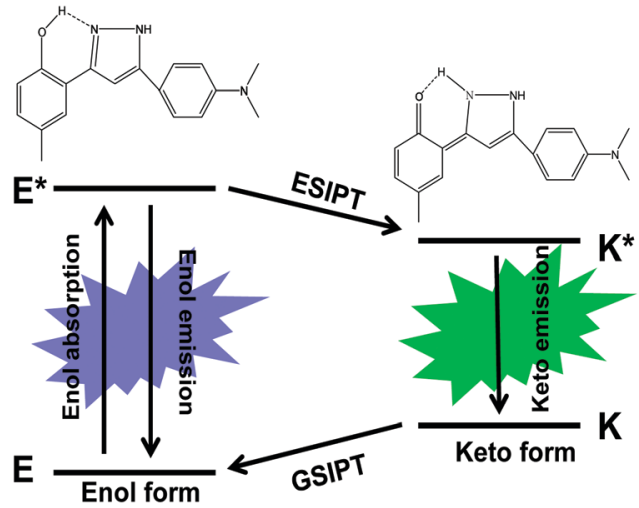

Scheme 1 Schematic representation of the ESIPT photo-cycle and chemical structures of the 4MPP enol form and keto form.

various stacking modes, which correspondingly influence their spectroscopic behaviours, especially the characteristics upon compression via a DAC device or under temperature. This study provides deeper understanding of the ESIPT materials and pave a successful way for effective control of the excited state transition between $\mathrm{E}^{*}$ and $\mathrm{K}^{*}$.

\section{Experimental section}

\subsection{Materials and measurements}

The targeted 4MPP crystals were obtained from Hongyu Zhang's group (Jilin University, China). The 4MPP materials were synthesized from 1,3-diaryl $\beta$-diketones and hydrazine hydrate, and the detailed synthetic procedure was introduced in detail in the reported literature. ${ }^{37}$ Two types of crystalline phases of 4MPP in this study are noted as GC (green crystal) and BVC (blue-violet crystal). The diffraction measurements of GC and BVC were performed on a Japan Science R-AXIS RAPID X-ray single crystal diffractometer. And their crystal structures were solved by direct methods using SHELXS-97 and refined on $F^{2}{ }^{38}$ Subsequently, the detailed analysis of molecular interaction and the stacking structures were based on Diamond software.

The high hydrostatic pressure conditions were produced by using a DAC (BGI-type) of $400 \mu \mathrm{m}$ culet diamonds. T301 stainless steel sheets of $250 \mu \mathrm{m}$ in thickness provide the gaskets with sample chambers of $200 \mu \mathrm{m}$ in diameter. Silicon oil has been used as the pressure-transmitting media in order to provide a guarantee for obtaining the hydrostatic pressure. ${ }^{39,40}$ The fluorescence of ruby loaded along with the sample was used as a standard for pressure. ${ }^{41,42}$

The high-pressure fluorescence spectra at room temperature were acquired on a fluorescence microscope (IX71, Olympus $20 \times$, numerical aperture $=0.4$ ) equipped with a spectrometer (Horiba Jobin Yvon iHR320), whose excitation wavelength is $365 \mathrm{~nm}$ produced from a light source of a mercury lamp. ${ }^{43}$ The measurement of the ruby chip was conducted on a Horiba Jobin Yvon T64000 Raman spectrometer with a $1800 \mathrm{~g} \mathrm{~mm}^{-1}$ holographic grating and the excitation wavelength from the argon ion laser is $514.5 \mathrm{~nm}$ line with $7 \mathrm{~mW}$ of output power. 
The data of high-pressure Raman spectra were acquired on a confocal Raman system which is based on an optical microscope (LabRAM Aramis, Horiba JobinYvon) used to focus the excitation light of $633 \mathrm{~nm}$ with the power of $7 \mathrm{~mW}$, a $50 \times / 0.75$ NA microscope objective, and a standard CCD array detector. All spectra were obtained with an integration time of $10 \mathrm{~s}$ under the same conditions if not specially pointed out.

The photoluminescence (PL) spectra with different temperatures were recorded on an Olympus IX73 microscope, equipped with an Andor spectrometer (SR-500i-D2-R) and a laser with the wavelength of $375 \mathrm{~nm}$. The range of temperature was from room temperature down to $-190{ }^{\circ} \mathrm{C}$ or up to $190{ }^{\circ} \mathrm{C}$ at a temperature gradient of $20{ }^{\circ} \mathrm{C}$ relying on a temperature-regulating device (LINKAM TS1500 + TMS94 + LNP94/2) with the cooling of liquid nitrogen.

\subsection{Computational methods}

Theoretical calculation of structures with pressures was carried out by using the CASTEP package in Material Studio 7. ${ }^{44,45}$ This calculation was performed with a plane wave set using Normconserving pseudopotentials ${ }^{46}$ with $750 \mathrm{eV}$ energy cutting off. In order to achieve geometry optimization, the energy tolerance was set as $2.0 \times 10^{-5} \mathrm{eV}$ per atom with a force tolerance of $0.05 \mathrm{eV} \AA^{-1}$, a maximum displacement of $2.0 \times 10^{-3} \AA$, and a maximum stress tolerance of $0.10 \mathrm{GPa}$. The energy tolerance was set as $1.0 \times 10^{-6} \mathrm{eV}$ per atom for the self-consistent field (SCF) calculation. The initial stacking modes and geometries based on the structure data of single-crystal diffraction were fully relaxed under external stress values of $0,1.0,2.0,3.0,4.0$, 5.0, 6.0, 7.0 and 8.0 GPa. The generalized gradient approximation (GGA) with Perdew Burke Ernzerhof $(\mathrm{PBE})^{47}$ was used to describe the exchange-correlation (XC) effects. TS scheme was used for dispersion corrections. And Hirshfeld surfaces (HSs) were generated using Crystal Explorer $3.1^{48,49}$ based on results of the crystal data of the optimized cell units obtained from Material Studio 7.

The calculated Raman spectrum of 4MPP at ambient pressure was optimized by using Becke's LYP (B3LYP) exchange-correlation functional with the 6-31G(d,p) basis set based on density functional theory (DFT). ${ }^{50,51}$ And the calculation of the Raman spectra at different pressures was attained on the basis of the structures obtained from the calculated results through Material Studio 7. The geometry of the 4MPP molecule was built with the Gaussian 09 package.

\section{Results and discussion}

\subsection{Single crystal structure}

The molecular conformation and intermolecular interactions may affect the ESIPT feature of 4MPP. Two polymorphs of 4MPP were attained under ambient conditions, but show clearly different photo-physical properties. As shown in Fig. 1a, GC appears green with the emission peak located at $536 \mathrm{~nm}\left(\mathrm{~K}^{*}\right)$, while BVC appears blue-violet with the emission band at $430 \mathrm{~nm}$ $\left(\mathrm{E}^{*}\right)$ and $616 \mathrm{~nm}\left(\mathrm{~K}^{*}\right)$, which demonstrates that two polymorphs

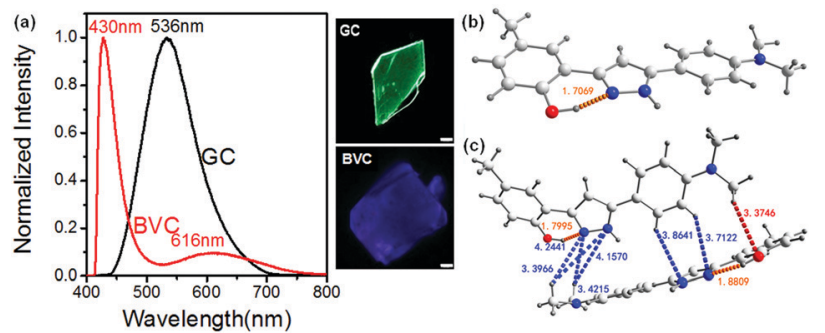

Fig. 1 Normalized fluorescence spectra and the corresponding photos of GC and BVC (a). The molecular conformations of GC (b) and BVC (c) under the excitation wavelength of $365 \mathrm{~nm}$. The scale is $50 \mu \mathrm{m}$.

go through different luminous paths (Scheme 1). The singlecrystal X-ray diffraction analysis of two crystals clearly indicates that their stacking mode seems like a fishbone shape (Fig. S1, ESI $\dagger$ ). An intramolecular hydrogen bond is one of the crucial elements for an efficient ESIPT process, which contains a hydroxyl group as a proton donor and a nitrogen-atom as an acceptor. For GC, the twisted angle of the $\mathrm{C}-\mathrm{C}-\mathrm{C}-\mathrm{N}$ is $5.575^{\circ}$ (Table S1, ESI $\dagger$ ), suggesting that GC is a nearly planar molecule that facilitates the transfer of $\mathrm{H}$ atoms in the excited state. ${ }^{52,53}$ In contrast, as for BVC in Fig. 1c, we can observe two kinds of molecular conformations, nearly planar and twisted ones, whereas two $\mathrm{N}, \mathrm{N}$-dimethyl-substituted aromatic groups are located in the opposite direction. The $\mathrm{C}-\mathrm{C}-\mathrm{C}-\mathrm{N}$ twisted angle of the twisted conformation in BVC is $20.708^{\circ}$, while the distance is $1.8809 \AA$, which is longer than GC (1.7069 $)$ ). Thus, the $\mathrm{H}$-transfer in BVC is restricted, leaving it in the normal emission $\left(\mathrm{E}^{*}\right)$, which confirms that strong intramolecular hydrogen bonding facilitates the ESIPT process over weak H-bonding. ${ }^{54}$ As a result, GC shows the emission from ESIPT $\left(\mathrm{K}^{*}\right)$ while BVC displays the normal emission ( $\mathrm{E}^{*}$ ). Fig. 1c and Table S2 (ESI $\dagger$ ) present the intermolecular interactions between the different conformations in BVC, such as $\mathrm{CH} / \mathrm{N}$ and $\mathrm{CH} / \mathrm{O}$. These intermolecular interactions make two different 4MPP molecules tightly close to each other in its crystal form, probably causing different behaviours from GC under external stimuli.

The stacking structures and intermolecular interactions were further analysed based on the results of a single crystal $\mathrm{X}$-ray diffractometer. In either GC or BVC, two 4MPP molecules are antiparallel and close to each other through weak $\pi / \pi$ intermolecular interactions between adjacent aromatic rings. The corresponding face-to-face distance between adjacent planes is $3.7749 \AA$ in GC, which is a little shorter than that in BVC (3.9428 ̊). The diversiform intermolecular interactions, such as $\mathrm{NH} / \mathrm{O}, \mathrm{OH} / \mathrm{N}, \mathrm{NH} / \pi, \mathrm{CH} / \mathrm{N}, \mathrm{CH} / \pi$ and $\mathrm{CH} / \mathrm{O}$, are highlighted in Fig. 2a (the details are summarized in Table S3, ESI $\dagger$ ). Differently, BVC only supports two representative intermolecular interactions, $\mathrm{CH} / \mathrm{N}$ and $\mathrm{CH} / \mathrm{O}$ (Fig. $2 \mathrm{~b}$ and Table S4, ESI $\dagger$ ), with the distances of 2.33-4.10 ̊. As is known to all, a hydrogen bond is a remarkable noncovalent driving force for ordered accumulation and has been widely used in crystal engineering. For GC shown in Fig. 2a, the intermolecular $\mathrm{N}-\mathrm{H} \cdots \mathrm{O}$ stands for a distance of $2.0934 \AA$, which is substantially larger than that of the intramolecular $\mathrm{O}-\mathrm{H} \cdots \mathrm{N}$ distance $(1.7065 \AA)$, indicating that the 

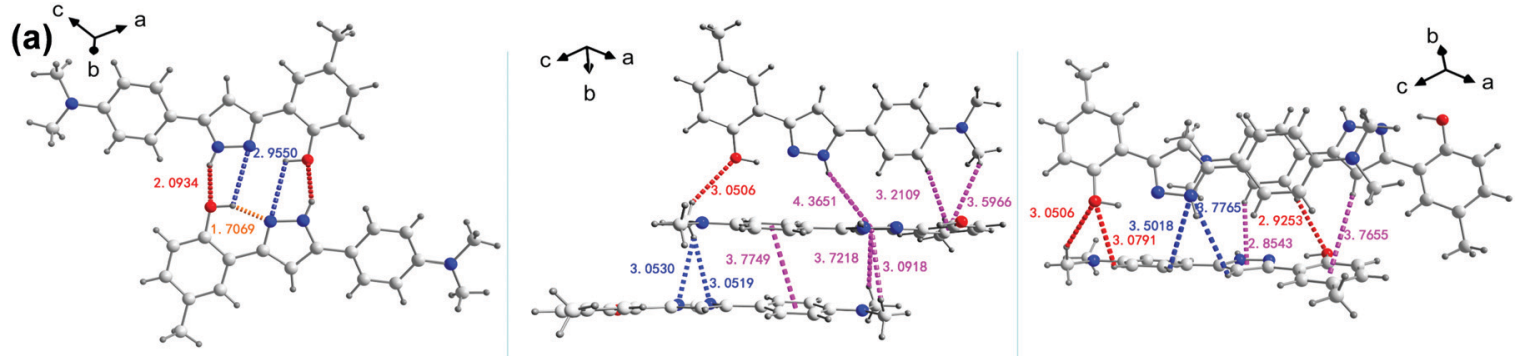

(b)
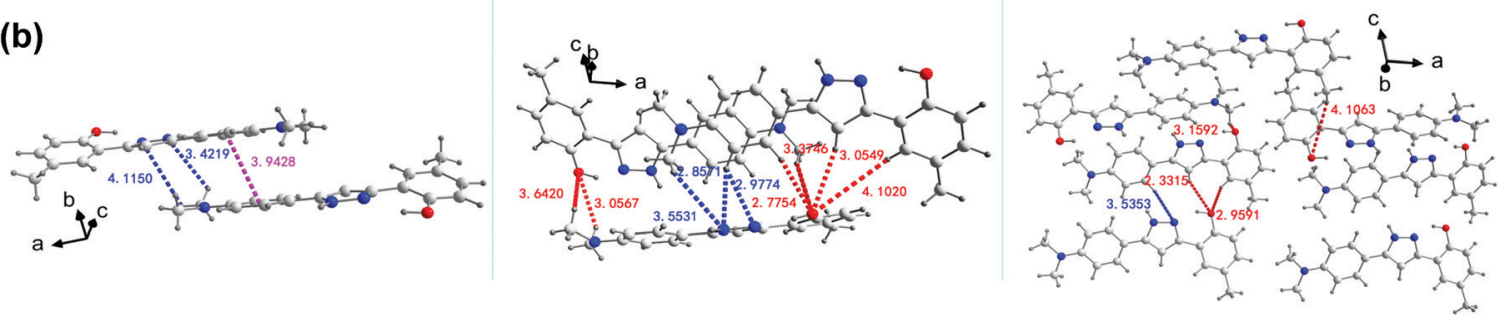

Fig. 2 The analysis of the intermolecular interactions in GC (a) and BVC (b), respectively (distance unit: Å).

intermolecular hydrogen bonds appear much weaker than the intramolecular hydrogen bonds, and thus the intermolecular proton transfer would be restricted. ${ }^{55,56}$ The intermolecular interactions and the strength of the hydrogen bonds varying with the distinct molecular packing modes can be a dominant factor in the polymorph-dependent luminescence, ${ }^{57}$ as for BVC and GC in the present study. Two 4MPP polymorphs with completely different molecular conformation, stacking structures and intermolecular interactions are expected to exhibit different fluorescent behaviours.

\subsection{Fluorescent behaviour under hydrostatic pressure}

In order to give further insights into the mechanism of PLMs and quantify the dependence of emission transformation on the external force, the pressure-dependent fluorescence spectra of two polymorphs were recorded via a DAC and contrastively analysed. As shown in Fig. 3a, the pristine GC crystal appears transparent and green, and has almost no identifiable colour changes during the pressurizing process, except the gradually weakened fluorescence resulting from the enhanced $\pi-\pi$ interactions and aggregation induced quenching. In the pressure-dependent fluorescence (a)

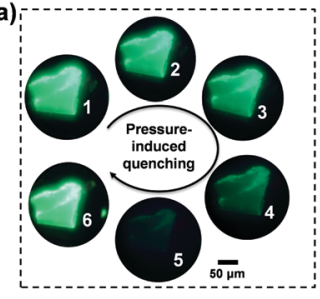

(b) 80,0
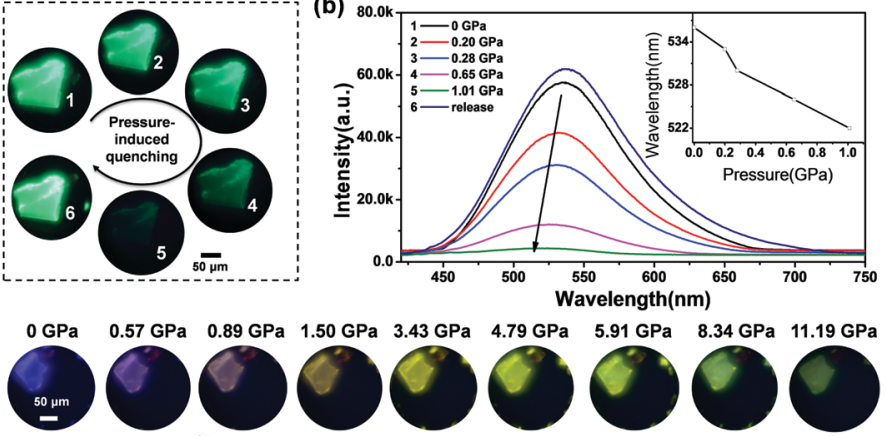

(d)

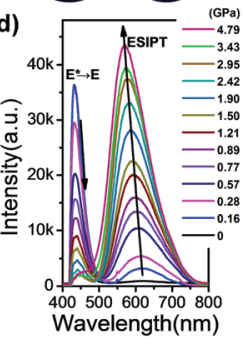

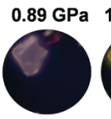
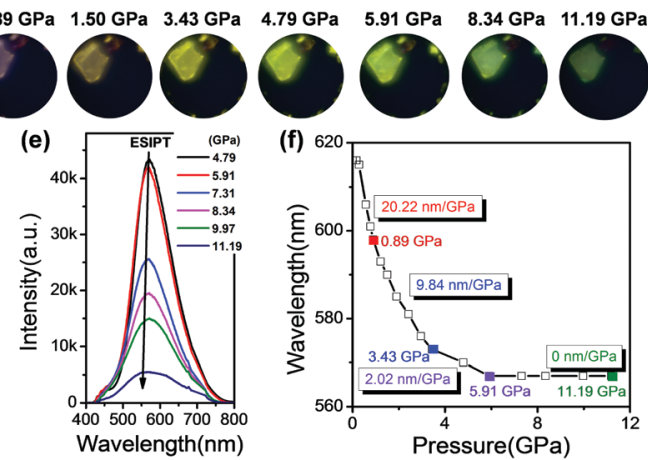

Fig. 3 (a) Fluorescent photos of GC at different pressure values and (b) corresponding fluorescence spectra of GC during compression and decompression via DAC and the inserted graph is the wavelength as a function of pressure. (c) Fluorescent photos of BVC at different pressure values. (d) Fluorescence spectra of BVC during compression from 0 to $4.79 \mathrm{GPa}$ via DAC. (e) Fluorescence spectra of BVC with further compression to $11.19 \mathrm{GPa}$. (f) Pressure-induced shifts of the emission band $(616 \mathrm{~nm})$ and the rate of blue-shifts at different stages during the compression. The scale is $50 \mu \mathrm{m}$. Excitation wavelength is $365 \mathrm{~nm}$. 
spectra of GC, a weak blue-shift of the emission band is observed in Fig. 3b. Previous literature reports have indicated that the blue-shift of the ESIPT materials during the conversion from a solution state to a frozen state is mainly explained by the formation of aggregates that suppress molecular motion in the rigid environments. ${ }^{58,59}$ So we speculate that the significant blue-shifts for GC during compression could be assigned to the continuously close-packing of molecules and the shortened intermolecular distances with increasing pressure. Also, the absorption spectra of GC show gradual red-shifts during the compression process (Fig. S2a, ESI $\dagger$ ), suggesting that the structure of GC approaches a more planarized structure with pressure along with a decrease in the cell volume, which will strengthen dipole-dipole interactions between two adjacent molecules during the compression, and cause the blue-shift of the emission band as well. ${ }^{60-62}$ The close-packing of molecules is thought to be propitious to the planar conformation of the $\mathrm{K}^{*}{ }^{58,63}$ Our data further support this conclusion. Different from GC, the fluorescent images of BVC show an identifiable colour evolution from blue-violet to yellow with hydrostatic pressure increasing (Fig. 3c). Initially, when the applied pressure increases from 0 to $4.79 \mathrm{GPa}$ (Fig. 3d), BVC turns into yellow gradually, accompanied by a transition of the emission band from 430 to $616 \mathrm{~nm}$ (a $186 \mathrm{~nm}$ red-shift) and a tiny blue-shift of the $616 \mathrm{~nm}$ band. These changes are considered to be from the conversion from $\mathrm{E}^{*}$ to $\mathrm{K}^{*}$, that is, the increased relative populations of the $\mathrm{K}^{*}$ state to the $\mathrm{E}^{*}$ state due to promotion of ESIPT caused by the planarization of the twisted conformation with the increased pressure. Previous publications indicated that the ESIPT emission depends on the stabilization of $\mathrm{K}^{*}$ through the effective delocalization of electrons in molecules, ${ }^{64-66}$ and the twisted molecular structure has a larger potential energy barrier height than the nearly planar one. So, the ESIPT process more easily occurs in the nearly planar structure rather than the twisted structure. ${ }^{62}$ Herein, the gradual planarized conformation of BVC leads to a stable six-membered ring-hydrogen bond transfer process, along with an increase in the conjugation degree and a decrease of the potential barrier, ${ }^{54}$ both of which facilitate the ESIPT process during the compression process. Also, it should be mentioned that the ESIPT process is activated by the enhanced $\mathrm{O}-\mathrm{H} \cdots \mathrm{N}$ intramolecular interactions, which will be discussed in detail later.

Moreover, to further prove the conformational planarization of 4MPP under high pressure, the absorption spectra of BVC under high pressure were recorded as well. As shown in Fig. S2b (ESI $\dagger$ ), the obvious red-shifts with increasing pressure are observed. The red-shifts in the absorption spectra indicate that the better delocalisation is caused by coplanar structure change of the 4MPP molecule during the compression process. Thereafter, when the pressure further rises, the emission band keeps unchanged, but the intensity gradually weakens (Fig. 3e), which is in agreement with the changes of GC. Additionally, in Fig. 3f, BVC shows obvious blue-shifts at different rate during the whole compression process. The rate of blue-shifts gradually decreases from $20.22 \mathrm{~nm} \mathrm{GPa}{ }^{-1}(0-0.89 \mathrm{GPa})$ to $9.84 \mathrm{GPa}$ (0.89-3.43 GPa) and then to $2.02 \mathrm{~nm} \mathrm{GPa}^{-1}(3.43-5.91 \mathrm{GPa})$, and finally the position of its emission maintains at $567 \mathrm{~nm}$ motionlessly even further increasing the pressure up to $11.19 \mathrm{GPa}$. This means that the transfer from $\mathrm{E}^{*}$ to $\mathrm{K}^{*}$ in the BVC system arrives at an equilibrium under such a pressure. Upon releasing the pressure, BVC shows incomplete recovery such that its luminescence restores to a light blue colour (Fig. S3, ESI $\dagger$ ), not the initial blue-violet crystal, which might be caused by the lattice disturbance under high pressure. ${ }^{26}$ Subsequently, a second pressurization was performed on the same sample, which experiences almost an identical varying tendency of the luminescence spectra as those during the first compression (Fig. S4, ESI $\dagger$ ).

\subsection{Raman spectra under hydrostatic pressure}

To further elucidate the mechafluorochromism at a molecular level, high-pressure Raman experiments for GC and BVC were also performed with an excitation wavelength of $633 \mathrm{~nm}$. The Raman spectra of GC and BVC show almost the same spectral profiles and their corresponding vibrational modes based on the calculated results are summarized in Table S5 (ESI $\dagger$ ). This discrimination of their features can be explained by their large difference in intermolecular interactions, where the peak at $3421 \mathrm{~cm}^{-1}$, assigned to the $\mathrm{N}-\mathrm{H}$ stretching vibration, only appears in BVC. In $\mathrm{GC}$, the $\mathrm{N}-\mathrm{H} \cdots \mathrm{O}$ intermolecular interactions between two 4MPP molecules restrict the $\mathrm{N}-\mathrm{H}$ stretching vibration. Also, compared to GC, BVC shows a clearer Raman peak at $3379 \mathrm{~cm}^{-1}$ attributed to $\mathrm{O}-\mathrm{H}$ stretching vibration. Vibrational frequencies and the intensity of the $\mathrm{O}-\mathrm{H}$ stretch depend on the potential shape of $\mathrm{O}-\mathrm{H} \cdots \mathrm{N}$. The intensity of the Raman band is dominated by the change in the molecular polarization. The planar GC has stronger intramolecular hydrogen bonds and the potential energy barrier in the doubleminimum potential becomes lower. As a result, the molecular polarization and the intensity of the Raman band both decrease, causing the weakened Raman intensities of GC. Fig. 4a and Fig. S5 (ESI $\dagger$ ) display the Raman spectra of GC at different pressures during compression. Most Raman peaks shift to higher wavenumbers due to the contraction of intermolecular distances and the shortened bond length. With further compression to $1.62 \mathrm{GPa}$, two modes at 951 (C-H wagging vibration out of plane) and $960 \mathrm{~cm}^{-1}$ (ring breathing vibration coupled with $\mathrm{C}-\mathrm{N}$ stretching vibration) start to merge into one, which might be caused by different shift-rate with the increased pressure. In particular, the frequency of $\mathrm{O}-\mathrm{H}$ stretching decreases from 3381 to $3372 \mathrm{~cm}^{-1}$ with the pressure up to $1.01 \mathrm{GPa}$, which is caused by the elongation of the $\mathrm{O}-\mathrm{H}$ bond involved in the intramolecular hydrogen bond. ${ }^{67,68}$ With further increasing pressure, the $3372 \mathrm{~cm}^{-1}$ band gradually disappears due to the gradual restricted vibration. Once the pressure is released, the Raman peaks are recovered and the final spectrum at normal pressure is in accordance with the original state, Fig. S6 (ESI $\dagger$ ), illustrating that the conformational changes are reversible. The Raman spectral evolution of BVC in DAC shows an obvious blueshift, which is coincident with the results of GC in Fig. S7 (ESI $\dagger$ ). It has been confirmed that BVC also owns reversible structural changes at a molecular level (Fig. S8, ESI $\dagger$ ). Significantly, the Raman bands at 3379 (the $\mathrm{O}-\mathrm{H}$ stretching vibration) and 


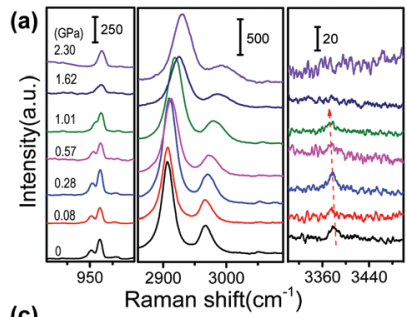

(c)
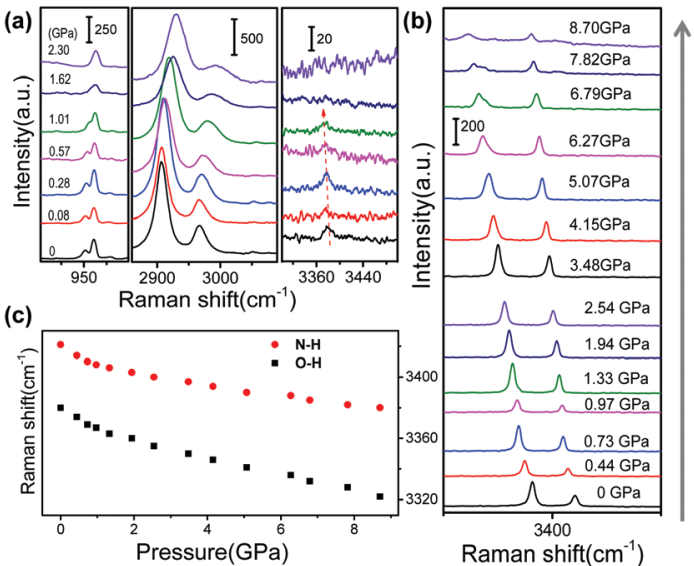

Fig. 4 Representative Raman patterns of GC (a) and BVC (b) at different pressure values applied by DAC. (c) The corresponding frequency shifts of $B V C$ as a function of pressure. Excitation wavelength is $633 \mathrm{~nm}$.

$3421 \mathrm{~cm}^{-1}$ (the $\mathrm{N}-\mathrm{H}$ stretching vibration) both show gradual red-shifts (Fig. 4b) during compression, indicating that the intramolecular hydrogen bond is gradually enhanced with a decrease of the distance of $\mathrm{H} \cdots \mathrm{N}$ and the elongation of the $\mathrm{O}-\mathrm{H}$ bond, ${ }^{56}$ which both facilitate the conversion from $\mathrm{E}^{*}$ to $\mathrm{K}^{*}$ in the excited state for BVC.

\subsection{Theoretical analysis of pressure-induced structural changes of BVC}

The theoretical calculations were also conducted based on the structure data in a unit cell of single crystal via Material Studio 7, which can provide deeper insights into the regulation of the BVC structures under compression. The optimized lattice parameters, the distance of $\mathrm{H} \cdots \mathrm{N}$ and the bond length of $\mathrm{O}-\mathrm{H}$ under the different external pressures ranging from 0 to 8.0 GPa are listed in Tables S6 and S7 (ESI $\dagger$ ). According to theoretical results, the pressure can significantly reduce the length of crystal axes $a, b$ and $c$ and the cell volume (Fig. 5a and b) as well, which makes the molecules stack more closely and certainly enhances the intermolecular interactions between neighbouring molecules. It should be noted that the cell volume of BVC as a function of pressure in Fig. 5b shows break points appearing at 1.0, 3.0 and 5.0 GPa, which is in agreement with different blue-shifting rate with pressure in the experiment (Fig. 4d), demonstrating that the blue-shifts of the emission band $\left(\mathrm{K}^{*}\right)$ of BVC are related to the molecular aggregation. Moreover, to investigate the change of the packing mode of BVC during the pressurization, the Hirshfeld surface was performed, because it can provide a direct three-dimensional view of intermolecular interactions based on the corresponding crystal structure at different pressures and already becomes a valuable tool for analysing intermolecular interactions. ${ }^{69}$ Hirshfeld surface mapped with the $d_{\text {norm }}$ of the 4MPP molecule based on the twisted and nearly planar conformations of 4MPP molecules in BVC are displayed in Fig. S9 and S10 (ESI $\dagger$ ). Obviously, more and more red regions appear on the surface of the 4MPP molecule (Fig. 5c) with pressure increasing, which indicates that the intermolecular interactions are enhanced by reducing the unit cell volume and the distance between neighbouring molecules under high pressure. Additionally, as shown in Fig. 5d and Table S7 (ESI $\dagger$ ), the distance of $\mathrm{H} \cdots \mathrm{N}$ is reduced and the bond length of $\mathrm{O}-\mathrm{H}$ is elongated with increasing pressure, which enhances the $\mathrm{O}-\mathrm{H} \cdots \mathrm{N}$ intramolecular interactions and finally facilitates the ESIPT process in the excited state. Moreover, the Raman spectra were calculated on the basis of theoretical results under different pressure values. Fig. 5e and Fig. S11 (ESI $\dagger$ ) show that most Raman bands shift to higher wavenumbers and two peaks assigned to $\mathrm{O}-\mathrm{H}$ and $\mathrm{N}-\mathrm{H}$ stretching vibrational mode have obvious red-shifts, in agreement with the high-pressure Raman results mentioned above.

Considering the consistency of the experimental and calculated results, we can infer the pressure-induced mechanism of BVC as follows: (1) pressure leads to 4MPP molecules stacking more intensely, as well as the enhancement of the intermolecular interactions. (2) Pressure also induces the contraction of distance of $\mathrm{H} \cdots \mathrm{N}$ and the elongation of the bond length of $\mathrm{O}-\mathrm{H}$, which both facilitate the ESIPT process in the excited state.

\subsection{Temperature-related photoluminescence spectroscopy}

Another notable feature of the present compounds is that they show remarkable colour changes with temperature, especially for GC. Fig. 6 shows the temperature-dependent fluorescence of GC and BVC, and Fig. S12 (ESI $\dagger$ ) shows their corresponding fluorescent pictures. In Fig. 6a, GC turns into blue with the temperature decreasing from 20 to $-190{ }^{\circ} \mathrm{C}$, and a conversion from $\mathrm{K}^{*}$ to $\mathrm{E}^{*}$ occurs, suggesting that the keto form is energetically unstable compared with the enol form. Thus, we can deduce that the ESIPT process is restricted with decreasing temperature. ${ }^{22}$ Also, the position of the peak in the fluorescence spectrum in Fig. S13a (ESI $\dagger$ ) shows a slight red-shift with further increasing temperature due to the increase in the cell volume with increasing temperature. $^{62}$ When it recovers to room temperature, the fluorescence spectrum is in agreement with the original emission profile (Fig. S13b, ESI $\dagger$ ), suggesting a reversible temperature response process of GC. In contrast, different from the discolouration behaviour of GC, BVC shows no obvious shifts and visible changes in its colour with the naked eye via temperature regulation. However, it is observed that the intensity of the $\mathrm{E}^{*}$ emission peak of BVC gradually decreases with increasing temperature (Fig. 6b), which can be explained by the thermal quenching of fluorescence. ${ }^{61,62}$ Interestingly, it should be noted that BVC suffers a phase transformation to GC until the temperature is increased up to $190{ }^{\circ} \mathrm{C}$ and it can retain its molecular conformation even when the temperature recovers to room temperature. This illustrates that the phase transformation is an irreversible process (Fig. 6c), further confirmed by their Raman spectra (Fig. S14, ESI $\dagger$ ), in which the Raman spectra of BVC, that is recovered from high temperature treatment, are identical to the spectral profile of GC. Furthermore, the corresponding X-ray diffraction (XRD) patterns and single crystal X-ray diffraction of GC, BVC and the BVC crystals recovered from $190{ }^{\circ} \mathrm{C}$ to room temperature, were also performed. The recovered XRD pattern (Fig. S15, ESI $\dagger$ ) and the single crystal parameters (Table S8, ESI $\dagger$ ) of BVC are consistent with GC, evidencing that the phase transformation with temperature occurs. 
(a)

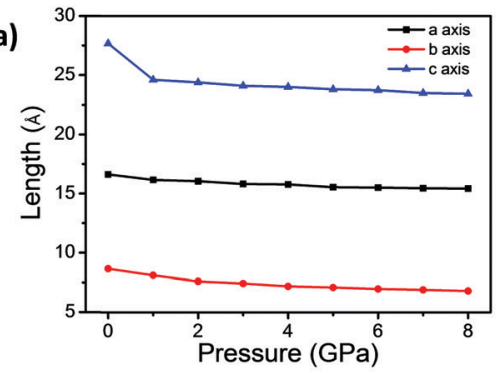

(c)
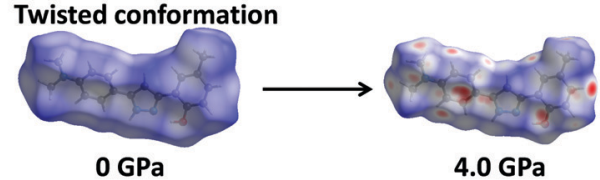

$4.0 \mathrm{GPa}$
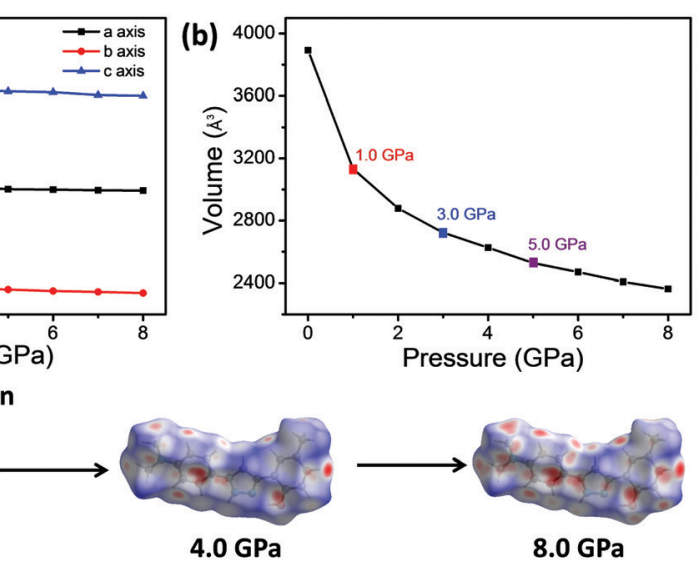

$8.0 \mathrm{GPa}$
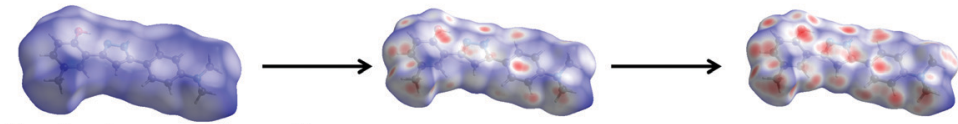

Nearly planar conformation
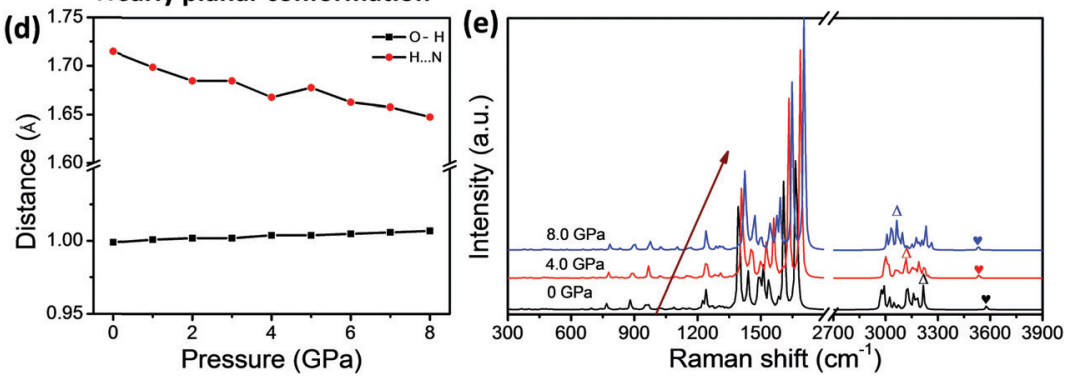

Fig. 5 Corresponding axial length (a) and cell volume (b) as a function of pressure based on the calculated results. (c) Hirshfeld surface of 4 MPP molecules based on twisted and nearly planar conformation in BVC under different pressure values of $0 \mathrm{GPa}, 4.0 \mathrm{GPa}$ and $8.0 \mathrm{Gpa}$. The red represents that the distance is shorter than the sum of van der Waals radii, while the white and blue indicate that the distance is longer than the sum of van der Waals radii. (d) Calculated pressure-dependent intramolecular hydrogen bond $\mathrm{O}-\mathrm{H} \cdots \mathrm{N}$ of 4 MPP based on twisted conformation in BVC. (e) Corresponding stimulated Raman spectra of the 4MPP molecule based on the twisted conformation in BVC under the external stress of $0 \mathrm{GPa}$, 4.0 GPa and 8.0 Gpa. The symbols " $\Delta$ " and " $"$ " represent the $\mathrm{O}-\mathrm{H}$ and $\mathrm{N}-\mathrm{H}$ stretching vibrational mode, respectively.
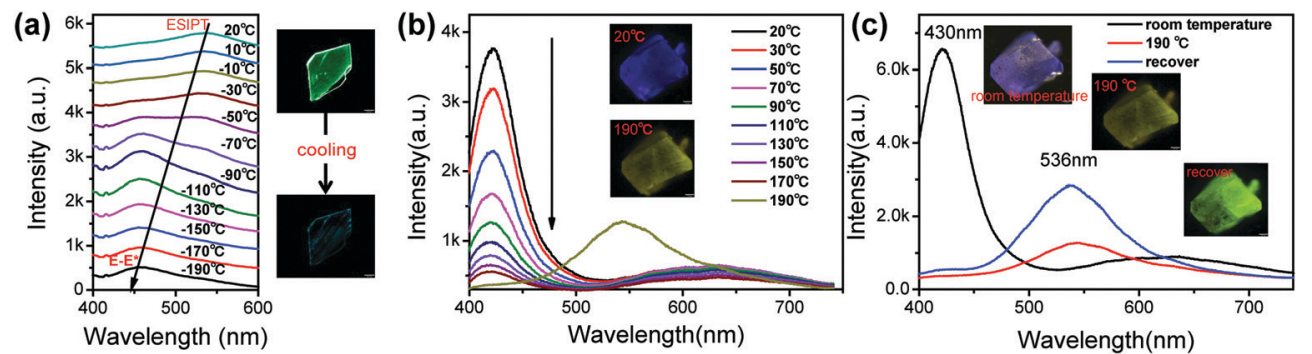

Fig. 6 Temperature-dependent fluorescence spectra of GC during $-190-20{ }^{\circ} \mathrm{C}$ (a), and BVC during $20-190{ }^{\circ} \mathrm{C}$ (b) and irreversible fluorescence switching with temperature up to $190^{\circ} \mathrm{C}$ (c). Excitation wavelength is $375 \mathrm{~nm}$.

\section{Conclusions}

In summary, we successfully realize the tunable switching of the state transition from $\mathrm{E}^{*}$ to $\mathrm{K}^{*}$ or $\mathrm{K}^{*}$ to $\mathrm{E}^{*}$ in $4 \mathrm{MPP}$ crystals for the first time. And a significant material system with ESIPT features is reported for understanding the mechanisms of structural conversion under pressure and temperature. The spectral behaviours of a multi-stimuli-responsive 4MPP in two polymorphs under pressure and temperature were compared, and the contrastive analysis reveals their ESIPT dynamics and photo-physical properties are remarkably different. GC could sense toward the pressure at $1.01 \mathrm{GPa}$, accompanied by unconspicuous blue-shifts and reduction in intensity. But, BVC undertakes an extrinsic pressure as high as $11.19 \mathrm{GPa}$ reversibly, along with tiny blue-shifts and visible colour changes, which are attributed to the conversion of $\mathrm{E}^{*}-\mathrm{K}^{*}$ caused by the molecular aggregation, planarity and enhanced intra-/inter-molecular interactions. Additionally, GC realized the reverse transition from ESIPT emission to normal emission with reduced temperature. Importantly, BVC suffered an abrupt and irreversible phase 
transformation to GC at high temperature. This study on 4MPP molecules provides a comprehensive insight into the origins of the properties of the stimuli-responsive luminescent materials based on the ESIPT mechanism, and also demonstrates that the ESIPT molecules can be potential candidates for applications in sensing and display devices with remarkable discolouration behaviours.

\section{Conflicts of interest}

There are no conflicts to declare.

\section{Acknowledgements}

This work was supported by the National Natural Science Foundation of China (NSFC 21573092, 21573087 and 21873039).

\section{Notes and references}

1 Y. Sagara and T. Kato, Nat. Chem., 2009, 1, 605-610.

2 Y. Sagara, S. Yamane, T. Mutai, K. Araki and T. Kato, Adv. Funct. Mater., 2009, 19, 1869-1875.

3 S. P. Anthony, Chem. - Asian J., 2012, 7, 374-379.

4 A. Kundu, P. S. Hariharan, K. Prabakaran, D. Moon and S. P. Anthony, Cryst. Growth Des., 2016, 16, 3400-3408.

5 J. E. Kwon and S. Y. Park, Adv. Mater., 2011, 23, 3615-3642.

6 B. Dick and N. P. Ernsting, J. Phys. Chem., 1987, 91, 4261-4265.

7 T. Iijima, A. Momotake, Y. Shinohara, T. Sato, Y. Nishimura and T. Arai, J. Phys. Chem. A, 2010, 5, 1603-1609.

8 J. Cheng, D. Liu, L. Bao, K. Xu, Y. Yang and K. Han, Chem. Asian J., 2014, 9, 3215-3220.

9 M. Pan, W. M. Liao, S. Y. Yin, S. S. Sun and C. Y. Su, Chem. Rev., 2018, 118, 8889-8935.

10 J. Goodman and L. E. Brus, J. Am. Chem. Soc., 1978, 22, 7472-7474.

11 S. K. Dogra, J. Photochem. Photobiol., A, 2005, 172, 196-206.

12 V. S. Padalkar and S. Seki, Chem. Soc. Rev., 2016, 45, 169-202.

13 A. Kundu, S. Karthikeyan, D. Moon and S. P. Anthony, ChemistrySelect, 2018, 3, 7340-7345.

14 Y. Hong, J. W. Y. Lam and B. Z. Tang, Chem. Soc. Rev., 2011, 40, 5361-5388.

15 Y. Liu, X. Tao, F. Wang, J. Shi, J. Sun, W. Yu, Y. Ren, D. Zou and M. Jiang, J. Phys. Chem. C, 2007, 111, 6544-6549.

16 T. Zhou, F. Li, Y. Fan, W. Song, X. Mu, H. Zhang and Y. Wang, Chem. Commun., 2009, 3199-3201.

17 J. Cheng, D. Liu, W. Li, L. Bao and K. Han, J. Phys. Chem. C, 2015, 119, 4242-4251.

18 L. Chen, S. Y. Yin, M. Pan, K. Wu, H. P. Wang, Y. N. Fan and C. Y. Su, J. Mater. Chem. C, 2016, 4, 6962-6966.

19 S. Y. Yin, S. S. Sun, M. Pan, L. Chen, Z. Wang, Y. J. Hou, Y. N. Fan, H. P. Wang and C. Y. Su, J. Photochem. Photobiol., A, 2018, 355, 377-381.

20 X. Mei, J. Wang, Z. Zhou, S. Wu, L. Huang, Z. Lin and Q. Ling, J. Mater. Chem. C, 2017, 5, 2135-2141.
21 A. Kundu, S. Karthikeyan, D. Moon and S. P. Anthony, CrystEngComm, 2017, 19, 6979-6985.

22 H. Konoshima, S. Nagao, I. Kiyota, K. Amimoto, N. Yamamoto, M. Sekine, M. Nakata, K. Furukawa and H. Sekiya, Phys. Chem. Chem. Phys., 2012, 14, 16448-16457.

23 P. Schattling, F. D. Jochum and P. Theato, Polym. Chem., 2014, 5, 25-36.

24 T. Han, Y. Zhang, X. Feng, Z. Lin, B. Tong, J. Shi, J. Zhi and Y. Dong, Chem. Commun., 2013, 49, 7049-7051.

25 P. Galer, R. C. Korosec, M. Vidmar and B. Sket, J. Am. Chem. Soc., 2014, 136, 7383-7394.

26 Y. Li, Z. Ma, A. Li, W. Xu, Y. Wang, H. Jiang, K. Wang, Y. Zhao and X. Jia, ACS Appl. Mater. Interfaces, 2017, 9, 8910-8918.

27 Z. Ma, Z. Wang, Y. Li, S. Song and X. Jia, Tetrahedron Lett., 2016, 57, 5377-5380.

28 Z. Ma, Z. Wang, X. Meng, Z. Ma, Z. Xu, Y. Ma and X. Jia, Angew. Chem., Int. Ed., 2016, 55, 519-522.

29 C. X. Chen, Z. W. Wei, Y. N. Fan, P. Y. Su, Y. Y. Ai, Q. F. Qiu, K. Wu, S. Y. Yin, M. Pan and C. Y. Su, Chem, 2018, 4, 1-12.

30 J. Wu, Y. Cheng, J. Lan, D. Wu, S. Qian, L. Yan, Z. He, X. Li, K. Wang, B. Zou and J. You, J. Am. Chem. Soc., 2016, 138, 12803-12812.

31 L. Wang, K. Wang, H. Zhang, C. Jiao, B. Zou, K. Ye, H. Zhang and Y. Wang, Chem. Commun., 2015, 51, 7701-7704.

32 Y. Dong, B. Xu, J. Zhang, X. Tan, L. Wang, J. Chen, H. Lv, S. Wen, B. Li, L. Ye, B. Zou and W. Tian, Angew. Chem., Int. Ed., 2012, 51, 10782-10785.

33 Q. Qi, J. Qian, X. Tan, J. Zhang, L. Wang, B. Xu, B. Zou and W. Tian, Adv. Funct. Mater., 2015, 25, 4005-4010.

34 S. Zhang, Y. Dai, S. Luo, Y. Gao, N. Gao, K. Wang, B. Zou, B. Yang and Y. Ma, Adv. Funct. Mater., 2017, 27, 1602276.

35 A. Li, Z. Ma, J. Wu, P. Li, H. Wang, Y. Geng, S. Xu, B. Yang, H. Zhang, H. Cui and W. Xu, Adv. Opt. Mater., 2018, 6, 1700647.

36 C. Feng, K. Wang, Y. Xu, L. Liu, B. Zou and P. Lu, Chem. Commun., 2016, 52, 3836-3839.

37 H. Liu, X. Cheng, Z. Y. Bian, K. Q. Ye and H. Y. Zhang, Chin. Chem. Lett., 2018, 29, 1537-1541.

38 G. M. Sheldrick and T. R. Schneider, Methods Enzymol., 1997, 277, 319-343.

39 J. M. Besson and J. P. Pinceaux, Rev. Sci. Instrum., 1979, 50, 541.

40 G. J. Piermarini, R. A. Forman and S. Block, Rev. Sci. Instrum., 1978, 49, 1061.

41 G. J. Piermarini and S. Block, Rev. Sci. Instrum., 1975, 46, 973-979.

42 J. H. Eggert, K. A. Goettel and I. F. Silvera, Phys. Rev. B: Condens. Matter Mater. Phys., 1989, 40, 5724-5732.

43 H. Li, H. Wang, D. Huang, L. Liang, Y. Gu, C. Liang, S. Xu and W. Xu, Rev. Sci. Instrum., 2014, 85, 056109.

44 B. Delley, J. Chem. Phys., 2000, 113, 7756-7764.

45 B. Delley, J. Chem. Phys., 1991, 94, 7245-7250.

46 J. P. Perdew, K. Burke and M. Ernzerhof, Phys. Rev. Lett., 1996, 77, 3865-3868.

47 G. B. Bachelet, D. R. Hamann and M. Schluter, Phys. Rev. B: Condens. Matter Mater. Phys., 1982, 26, 4199-4228. 
48 L. Sun, Y. Zhao, Y. Shang, C. Sun and M. Zhou, Chem. Phys. Lett., 2017, 689, 56-61.

49 J. J. McKinnon, D. Jayatilaka and M. A. Spackman, Chem. Commun., 2007, 3814-3816.

50 P. Hohenberg and W. Kohn, Phys. Rev., 1964, 136, B864-B871.

51 W. Kohn and L. J. Sham, Phys. Rev., 1965, 140, A1133-A1138.

52 P. Dongare, A. G. Bonn, S. Maji and L. Hammarström, J. Phys. Chem. C, 2017, 121, 12569-12576.

53 T. Mutai, H. Tomoda, T. Ohkawa, Y. Yabe and K. Araki, Angew. Chem., Int. Ed., 2008, 47, 9522-9524.

54 K. Furukawa, N. Yamamoto, T. Nakabayashi, N. Ohta, K. Amimoto and H. Sekiya, Chem. Phys. Lett., 2012, 539-540, 45-49.

55 J. E. Kwon and S. Y. Park, Adv. Mater., 2011, 23, 3615-3642.

56 Q. Wu, Q. Peng, Y. Niu, X. Gao and Z. Shuai, J. Phys. Chem. A, 2012, 116, 3881-3888.

57 T. Mutai, H. Shono, Y. Shigemitsu and K. Araki, CrystEngComm, 2014, 16, 3890-3895.

58 T. Mutai, H. Sawatani, T. Shida, H. Shono and K. Araki, J. Org. Chem., 2013, 78, 2482-2489.

59 V. S. Padalkar, D. Sakamaki, K. Kuwada, A. Horio, H. Okamoto, N. Tohnai, T. Akutagawa, K. Sakai and S. Seki, Asian J. Org. Chem., 2016, 5, 938-945.
60 K. Furukawa, K. Hino, N. Yamamoto, K. Awasthi, T. Nakabayashi, N. Ohta and H. Sekiya, J. Phys. Chem. A, 2015, 119, 9599-9608.

61 K. Furukawa, N. Yamamoto, K. Hino and H. Sekiya, Chem. Phys. Lett., 2016, 643, 109-113.

62 K. Furukawa, N. Yamamoto, K. Hino and H. Sekiya, Phys. Chem. Chem. Phys., 2016, 18, 28564-28575.

63 A. Douhal, F. Amat-Guerri and A. U. Acufia, J. Phys. Chem., 1995, 99, 76-80.

64 Y. Shigemitsu, T. Mutai, H. Houjou and K. Araki, Phys. Chem. Chem. Phys., 2014, 16, 14388-14395.

65 S. Nagaoka and U. Nagashima, J. Phys. Chem., 1988, 92, 166-171.

66 Y. Shigemitsu, T. Mutai, H. Houjou and K. Araki, J. Phys. Chem. A, 2012, 116, 12041-12048.

67 K. Wang, D. Duan, R. Wang, A. Lin, Q. Cui, B. Liu, T. Cui, B. Zou, X. Zhang, J. Hu, G. Zou and H. K. Mao, Langmuir, 2009, 25, 4787-4791.

68 S. Trudel and D. F. R. Gilson, Inorg. Chem., 2003, 42, 2814-2816.

69 M. A. Spackman and D. Jayatilaka, CrystEngComm, 2009, 11, 19-32. 